\title{
THE SEMICLASSICAL LIMIT OF EIGENFUNCTIONS OF THE SCHRÖDINGER EQUATION AND THE BOHR-SOMMERFELD QUANTIZATION CONDITION, REVISITED
}

\author{
D. R. YAFAEV
}

To Vasiliu Mikhailovich Babich on his 80th birthday

\begin{abstract}
The semiclassical limit, as the Planck constant $\hbar$ tends to 0 , of bound states of a quantum particle in a one-dimensional potential well is considered. The semiclassical asymptotic formulas for eigenfunctions are justified, and the Bohr-Sommerfeld quantization condition is recovered.
\end{abstract}

\section{§1. INTRODUCTION}

1.1. We study the limit as $\hbar \rightarrow 0$ of eigenfunctions $\psi(x)=\psi(x ; \lambda, \hbar)$ of the Schrödinger equation

$$
-\hbar^{2} \psi^{\prime \prime}(x)+v(x) \psi(x)=\lambda \psi(x), \quad v(x)=\overline{v(x)}, \quad \psi \in L^{2}(\mathbb{R}),
$$

for $\lambda$ close to some noncritical energy $\lambda_{0}$ (i.e., $v^{\prime}(x) \neq 0$ for $x$ such that $v(x)=\lambda_{0}$ ). We assume that the equation $v(x)=\lambda$ has exactly two solutions (the turning points) $x_{ \pm}=x_{ \pm}(\lambda)$ and that $v(x)<\lambda$ for $x \in\left(x_{-}, x_{+}\right)$. Thus, $\left(x_{-}, x_{+}\right)$is a potential well and the energy $\lambda$ is separated from its bottom. We suppose that the eigenfunctions $\psi(x)$ are real and normalized, that is,

$$
\int_{-\infty}^{\infty} \psi^{2}(x) d x=1
$$

It is a common wisdom that the limit of $\psi(x)=\psi(x ; \lambda, \hbar)$ as $\hbar \rightarrow 0$ is described by the Green-Liouville approximation away from the turning points $x_{ \pm}$. In neighborhoods of the turning points the asymptotic behavior of $\psi(x)$ is more complicated and is given in terms of an Airy function. Surprisingly, we have not found a precise formulation and a proof of this result in the literature. Our goal is to fill in this gap. Here we follow the method suggested by R. E. Langer and thoroughly exposed by F. W. J. Olver in his book 7 .

The detailed asymptotic behavior of $\psi(x)$ described in Theorems 2.5 and 4.4 allows one to recover the classical Bohr-Sommerfeld quantization condition on $\lambda$ (see Theorem 4.1). Actually, we prove somewhat more, establishing a one-to-one correspondence between the eigenvalues of the Schrödinger operator $H_{\hbar}=-\hbar^{2} d^{2} / d x^{2}+v(x)$ from a neighborhood of a noncritical energy and the points $(n+1 / 2) \hbar$, where $n$ is an integer. This implies the semiclassical Weyl formula for the distribution of eigenvalues of the operator $H_{\hbar}$ as $\hbar \rightarrow 0$, with a strong estimate of the remainder. It turns out (see Corollary 4.2) that this

2010 Mathematics Subject Classification. Primary 47A40, 81U05.

Key words and phrases. Schrödinger equation, potential well, Airy functions, Green-Liouville approximation, Bohr-Sommerfeld quantization condition, semiclassical Weyl formula.

Partially supported by the project NONAa, ANR-08-BLANC-0228. 
remainder never exceeds 1. Also, in Theorem 5.2, we obtain the quantization condition for discontinuous functions $v(x)$. This formula generalizes that of Bohr and Sommerfeld and is probably new.

We note that the Bohr-Sommerfeld quantization condition is also well known in much more difficult multidimensional problems. In this context we mention the book [1] by Babich and Buldyrev (where the ray approximation was used), the book $[3]$ by Fedoryuk and Maslov (where the Maslov canonical operator was used), and also the papers [5] by Helffer and Robert and 4 by Helffer, Martinez, and Robert (where the methods of microlocal analysis were used).

However, in the one-dimensional problem it is more natural to lean upon methods of ordinary differential equations. Such an approach was developed by Fedoryuk (see his book [2]) for analytic potentials. In this case the study of turning points can be avoided, so that the Airy function does not appear.

1.2. The asymptotic expansion of eigenfunctions yields (see Proposition 4.5) that of the observables

$$
\int_{-\infty}^{\infty} w(x) \psi^{2}(x ; \lambda, \hbar) d x
$$

for fairly arbitrary functions $w(x)$. For example, for $w(x)$ we can take characteristic functions of Borel subsets of $\mathbb{R}$ or choose $w(x)=v(x)$. This gives the asymptotic expansion of the kinetic energy

$$
K(\lambda, \hbar):=\hbar^{2} \int_{-\infty}^{\infty} \psi^{\prime}(x ; \lambda, \hbar)^{2} d x=K_{\mathrm{cl}}(\lambda)+O\left(\hbar^{1 / 3}\right)
$$

as $\hbar \rightarrow 0$ uniformly for $\lambda$ in a neighborhood of the point $\lambda_{0}$. The leading term $K_{\mathrm{cl}}(\lambda)$ (the index "cl" stands of course for the corresponding classical object) is given by the expression

$$
K_{\mathrm{cl}}(\lambda)=\int_{x_{-}(\lambda)}^{x_{+}(\lambda)}(\lambda-v(x))^{1 / 2} d x\left(\int_{x_{-}(\lambda)}^{x_{+}(\lambda)}(\lambda-v(x))^{-1 / 2} d x\right)^{-1}
$$

Note that the integrals here are taken over the classically allowed region, and that $K_{\mathrm{cl}}(\lambda)$ coincides (see Subsection 4.3) with the averaged value of the kinetic energy of a particle of energy $\lambda$ in classical mechanics.

We emphasize that our derivation of the quantization condition and of asymptotic formulas for the observables (1.2) requires Airy functions, although they do not enter the final answer. However, we do not know how to avoid Airy functions without additional assumptions on $v(x)$.

\section{§2. Semiclassical solutions of the Schrödinger Equation}

2.1. It is convenient to rewrite equation (1.1) as

$$
-u_{\hbar}^{\prime \prime}(x)+\hbar^{-2} q(x) u_{\hbar}(x)=0,
$$

where

$$
q(x)=q(x ; \lambda)=v(x)-\lambda .
$$

We need some regularity of the function $v(x)$ and a weak condition on its behavior at infinity.

Assumption 2.1. The function $v$ is of class $C^{2}(\mathbb{R})$ and, for some $\rho_{0}>1$, the function

$$
\left.\left.\left(|q(x)|^{-3} q^{\prime}(x)^{2}+q(x)^{-2}\left|q^{\prime \prime}(x)\right|\right)\left|\int_{0}^{x}\right| q(y)\right|^{1 / 2} d y\right|^{\rho_{0}}
$$

is bounded for sufficiently large $|x|$. 
The latter condition is satisfied in all reasonable cases. For example, if $v(x) \rightarrow v_{0}>\lambda$, it suffices to require that

$$
v^{\prime}(x)^{2}+\left|v^{\prime \prime}(x)\right|=O\left(|x|^{-\rho_{0}}\right), \quad \rho_{0}>1, \quad|x| \rightarrow \infty .
$$

It is also satisfied if $v(x)$ behaves at infinity as $|x|^{\alpha}$ or $e^{\alpha|x|}$ with $\alpha>0$; in these cases, $\rho_{0}=2$.

We consider the case of one potential well. To be more precise, we make the following assumption.

Assumption 2.2. The equation $v(x)=\lambda$ has two solutions $x_{+}=x_{+}(\lambda)$ and $x_{-}=$ $x_{-}(\lambda)$. We suppose that $x_{-}<x_{+}, v(x)<\lambda$ for $x \in\left(x_{-}, x_{+}\right), v(x)>\lambda$ for $x \notin\left[x_{-}, x_{+}\right]$, and

$$
\liminf _{|x| \rightarrow \infty} v(x)>\lambda
$$

Moreover, the function $v$ belongs to the class $C^{3}$ in some neighborhoods of the points $x_{ \pm}$and $\pm v^{\prime}\left(x_{ \pm}\right)>0$.

Note that if Assumption 2.2 is satisfied for some $\lambda_{0}$, then it is also satisfied for all $\lambda$ in some neighborhood of $\lambda_{0}$.

Our goal in this section is to describe the asymptotic properties as $\hbar \rightarrow 0$ of solutions $u_{+}(x)=u_{+}(x ; \lambda, \hbar)$ and $u_{-}(x)=u_{-}(x ; \lambda, \hbar)$ of equation (2.1) exponentially decaying as $x \rightarrow+\infty$ and $x \rightarrow-\infty$, respectively. These asymptotic properties will be given in terms of an Airy function and are uniform with respect to $x \in\left[x_{1}, \infty\right)$ or $x \in\left(-\infty, x_{1}\right]$, where $x_{1}$ is an arbitrary point of the interval $\left(x_{-}, x_{+}\right)$.

2.2. We recall the definition of the Airy functions and their properties required here (see, e.g., 7] for the details). Consider the equation

$$
-w^{\prime \prime}(t)+t w(t)=0
$$

and denote by $\mathrm{Ai}(t)$ its solution with the asymptotic formula

$$
\operatorname{Ai}(t)=2^{-1} \pi^{-1 / 2} t^{-1 / 4} \exp \left(-2 t^{3 / 2} / 3\right)\left(1+O\left(t^{-3 / 2}\right)\right), \quad t \rightarrow+\infty .
$$

Then

$$
\operatorname{Ai}(t)=\pi^{-1 / 2}|t|^{-1 / 4} \sin \left(2|t|^{3 / 2} / 3+\pi / 4\right)+O\left(|t|^{-7 / 4}\right), \quad t \rightarrow-\infty .
$$

Note that $\operatorname{Ai}(t)>0$ for all $t \geq 0$.

The solution $\mathrm{Bi}(t)$ of equation (2.2) is determined by its asymptotic behavior as $t \rightarrow-\infty$, which differs from (2.4) only by the phase shift:

$$
\operatorname{Bi}(t)=-\pi^{-1 / 2}|t|^{-1 / 4} \sin \left(2|t|^{3 / 2} / 3-\pi / 4\right)+O\left(|t|^{-7 / 4}\right), \quad t \rightarrow-\infty .
$$

For $t \geq 0$, this function is positive and satisfies the estimate

$$
\operatorname{Bi}(t) \leq C(1+t)^{-1 / 4} \exp \left(2 t^{3 / 2} / 3\right) .
$$

Here and below we denote by $C$ and $c$ various positive constants whose precise values are of no importance.

We also use the fact that all asymptotics (2.3), (2.4), and (2.5) can be differentiated in $t$. In particular, for the Wronskian we have

$$
\left\{\mathrm{Ai}(t), \mathrm{Bi}_{-}(t)\right\}:=\mathrm{Ai}^{\prime}(t) \mathrm{Bi}(t)-\mathrm{Ai}(t) \mathrm{Bi}^{\prime}(t)=-\pi^{-1} .
$$

It follows that

$$
\operatorname{Bi}(s) \mathrm{Ai}^{-1}(s)-\mathrm{Bi}(t) \mathrm{Ai}^{-1}(t)=\pi^{-1} \int_{t}^{s} \operatorname{Ai}^{-2}(\tau) d \tau, \quad s \geq t \geq 0 .
$$


2.3. To formulate results, we need the following auxiliary functions $\xi_{ \pm}(x)=\xi_{ \pm}(x ; \lambda)$ :

$$
\begin{aligned}
& \xi_{+}(x)=\left(\frac{3}{2} \int_{x_{+}}^{x} q(y)^{1 / 2} d y\right)^{2 / 3}, \quad x \geq x_{+}, \\
& \xi_{+}(x)=-\left(\frac{3}{2} \int_{x}^{x_{+}}|q(y)|^{1 / 2} d y\right)^{2 / 3}, \quad x_{-}<x \leq x_{+},
\end{aligned}
$$

and

$$
\begin{aligned}
& \xi_{-}(x)=\left(\frac{3}{2} \int_{x}^{x_{-}} q(y)^{1 / 2} d y\right)^{2 / 3}, \quad x \leq x_{-}, \\
& \xi_{-}(x)=-\left(\frac{3}{2} \int_{x_{-}}^{x}|q(y)|^{1 / 2} d y\right)^{2 / 3}, \quad x_{-} \leq x<x_{+} .
\end{aligned}
$$

Here is a list of properties of these functions. The following result is practically the same as Lemma 3.1 in [7, Chapter 11].

Lemma 2.3. Let $x_{1} \in\left(x_{-}, x_{+}\right)$. Then $\xi_{+} \in C^{3}\left(x_{1}, \infty\right), \xi_{-} \in C^{3}\left(-\infty, x_{1}\right)$, and $\xi_{ \pm}(x) \rightarrow$ $+\infty$ as $x \rightarrow \pm \infty$. The derivatives

$$
\pm \xi_{ \pm}^{\prime}(x)>0, \quad \xi_{ \pm}^{\prime}\left(x_{ \pm}\right)= \pm\left|v^{\prime}\left(x_{ \pm}\right)\right|^{1 / 3}
$$

and the functions $\xi_{ \pm}(x)$ satisfy the equation

$$
\xi_{ \pm}^{\prime}(x)^{2} \xi_{ \pm}(x)=q(x) .
$$

This lemma implies that the function

$$
p_{ \pm}(x)=\left(\left|\xi_{ \pm}^{\prime}(x)\right|^{-1 / 2}\right)^{\prime \prime}\left|\xi_{ \pm}^{\prime}(x)\right|^{-3 / 2}
$$

is continuous. Moreover, using identity (2.10), we see that

$$
-16 p_{ \pm}(x)=5 \xi_{ \pm}(x)^{-2}+\xi_{ \pm}(x)\left(4 q(x)^{-2} q^{\prime \prime}(x)-5 q(x)^{-3} q^{\prime}(x)^{2}\right), \quad x \neq x_{ \pm},
$$

whence, in accordance with Assumption 2.1,

$$
\left|p_{ \pm}(x)\right| \leq C\left|\xi_{ \pm}(x)\right|^{-1 / 2-\rho}, \quad \rho=3 \min \left\{\rho_{0}-1,1\right\} / 2>0 .
$$

2.4. We construct solutions $u_{ \pm}(x)=u_{ \pm}(x ; \lambda, \hbar)$ of equation (2.1) with semiclassical asymptotic behavior as $\hbar \rightarrow 0$ or (and) $x \rightarrow \pm \infty$. We define these solutions by their asymptotic formulas as $x \rightarrow \pm \infty$. Below all asymptotic relations are assumed to be differentiable with respect to $x$. In this subsection, we only formulate results.

Proposition 2.4. Under Assumption 2.1, for every fixed $\hbar>0$, equation (2.1) has a (unique) solution $u_{ \pm}(x)$ such that

$$
\begin{array}{r}
u_{ \pm}(x)=2^{-1} \pi^{1 / 2} \hbar^{1 / 6} q(x)^{-1 / 4} \exp \left(\mp \hbar^{-1} \int_{x_{ \pm}}^{x} q(y)^{1 / 2} d y\right) \\
\times\left(1+O\left(\left|\int_{x_{ \pm}}^{x} q(y)^{1 / 2} d y\right|^{-\rho_{1}}\right)\right),
\end{array}
$$

where $\rho_{1}=\min \left\{\rho_{0}-1,1\right\}>0$ as $x \rightarrow \pm \infty$.

Uniform asymptotic formulas for $u_{ \pm}(x)$ are given in the following assertion.

Theorem 2.5. Let Assumptions 2.1 and 2.2 hold. If $\pm x \geq \pm x_{ \pm}$, then the solutions $u_{ \pm}(x)=u_{ \pm}(x ; \lambda, \hbar)$ admit the representations

$$
u_{ \pm}(x)=\left|\xi_{ \pm}^{\prime}(x)\right|^{-1 / 2} \operatorname{Ai}\left(\hbar^{-2 / 3} \xi_{ \pm}(x)\right)\left(1+\varepsilon_{ \pm}(x ; \hbar)\right),
$$


where the remainder satisfies the estimate

$$
\left|\varepsilon_{ \pm}(x ; \lambda, \hbar)\right| \leq C \hbar\left(1+\left|\xi_{ \pm}(x)\right|\right)^{-\rho}, \quad \rho=3 \min \left\{\rho_{0}-1,1\right\} / 2>0 .
$$

Let $x_{1} \in\left(x_{-}, x_{+}\right)$. On the interval $\left[x_{1}, x_{+}\right]$(on the interval $\left[x_{-}, x_{1}\right]$ ) the function $u_{+}$ (the function $u_{-}$) admits the representation

$$
u_{ \pm}(x)=\left|\xi_{ \pm}^{\prime}(x)\right|^{-1 / 2} \mathrm{Ai}\left(\hbar^{-2 / 3} \xi_{ \pm}(x)\right)+O\left(\hbar^{7 / 6}\left(\hbar^{2 / 3}+\left|x-x_{ \pm}\right|\right)^{-1 / 4}\right) .
$$

Away from the points $x_{ \pm}$, we can replace the Airy function $\mathrm{Ai}(t)$ by its asymptotic formulas (2.3) or (2.4). Indeed, by (2.9), we have

$$
\left|\xi_{ \pm}(x)\right| \geq c\left|x-x_{ \pm}\right|, \quad c>0
$$

whence $\quad \hbar^{-2 / 3} \xi_{+}(x) \rightarrow \pm \infty \quad$ if $\quad \hbar^{-2 / 3}\left(x-x_{+}\right) \rightarrow \pm \infty \quad$ and $\quad \hbar^{-2 / 3} \xi_{-}(x) \rightarrow \pm \infty \quad$ if $\hbar^{-2 / 3}\left(x-x_{-}\right) \rightarrow \mp \infty$. This leads to the following result.

Corollary 2.6. Suppose that $\delta_{\hbar} \hbar^{-2 / 3} \geq c>0$ (in particular, $\delta_{\hbar}$ may be fixed). Then the functions $u_{ \pm}(x)$ have the following asymptotic formulas :

$$
u_{ \pm}(x)=2^{-1} \pi^{1 / 2} \hbar^{1 / 6} q(x)^{-1 / 4} \exp \left(\mp \hbar^{-1} \int_{x_{ \pm}}^{x} q(y)^{1 / 2} d y\right)\left(1+O\left(\hbar\left|\xi_{ \pm}(x)\right|^{-3 / 2}\right)\right)
$$

as $\hbar \rightarrow 0$, uniformly in $x \geq x_{+}+\delta_{\hbar}$ for $u_{+}(x)$ and in $x \leq x_{-}-\delta_{\hbar}$ for $u_{-}(x)$. Let $x_{1} \in\left(x_{-}, x_{+}\right)$. Then the functions $u_{ \pm}(x)$ have the following asymptotic formulas:

$$
\begin{array}{r}
u_{ \pm}(x)=\pi^{1 / 2} \hbar^{1 / 6}|q(x)|^{-1 / 4} \sin \left( \pm \hbar^{-1} \int_{x}^{x_{ \pm}}|q(y)|^{1 / 2} d y+\pi / 4\right) \\
+O\left(\hbar^{7 / 6}\left|x-x_{ \pm}\right|^{-7 / 4}\right)
\end{array}
$$

as $\hbar \rightarrow 0$, uniformly in $x \in\left[x_{1}, x_{+}-\delta_{\hbar}\right]$ for $u_{+}(x)$ and uniformly in $x \in\left[x_{-}+\delta_{\hbar}, x_{1}\right]$ for $u_{-}(x)$.

On the other hand, using (2.17) and the estimate $|\mathrm{Ai}(t)| \leq C(1+|t|)^{-1 / 4}$, we obtain estimates, uniform in $\hbar$, of the functions $u_{ \pm}(x)$ in neighborhoods of the turning points.

Corollary 2.7. For sufficiently small $\left|x-x_{ \pm}\right|$, we have the estimate

$$
\left|u_{ \pm}(x)\right| \leq C\left(1+\hbar^{-2 / 3}\left|x-x_{ \pm}\right|\right)^{-1 / 4}
$$

with a constant $C$ independent of $\hbar$.

We note that all asymptotic relations (2.14), (2.16), (2.18), and (2.19) can be differentiated with respect to $x$. In particular, we have the asymptotic formulas

$$
\begin{array}{r}
u_{ \pm}^{\prime}(x)=\mp \pi^{1 / 2} \hbar^{-5 / 6}|q(x)|^{1 / 4} \cos \left( \pm \hbar^{-1} \int_{x}^{x_{ \pm}}|q(y)|^{1 / 2} d y+\pi / 4\right) \\
+O\left(\hbar^{1 / 6}\left|x-x_{ \pm}\right|^{-7 / 4}\right)
\end{array}
$$

as $\hbar \rightarrow 0$, uniformly in $x \in\left[x_{1}, x_{+}-\delta_{\hbar}\right]$ for $u_{+}(x)$ and uniformly in $x \in\left[x_{-}+\delta_{\hbar}, x_{1}\right]$ for $u_{-}(x)$. All these relations can also be differentiated with respect to $\lambda$. For example, we have

$$
\begin{aligned}
\partial u_{ \pm}(x ; \lambda, \hbar) / \partial \lambda= & \pm 2^{-1} \pi^{1 / 2} \hbar^{-5 / 6}|q(x ; \lambda)|^{-1 / 4} \int_{x}^{x_{ \pm}}|q(y ; \lambda)|^{-1 / 2} d y \\
& \times \cos \left( \pm \hbar^{-1} \int_{x}^{x_{ \pm}}|q(y ; \lambda)|^{1 / 2} d y+\pi / 4\right)+O\left(\hbar^{1 / 6}\left|x-x_{ \pm}\right|^{-7 / 4}\right)
\end{aligned}
$$

as $\hbar \rightarrow 0$, uniformly in $x \in\left[x_{1}, x_{+}-\delta_{\hbar}\right]$ for $u_{+}(x)$ and uniformly in $x \in\left[x_{-}+\delta_{\hbar}, x_{1}\right]$ for $u_{-}(x)$. 
2.5. Now we calculate the norm of the function $u_{ \pm}(x)$ in the space $L^{2}\left(x_{1}, \pm \infty\right)$, where $x_{1} \in\left(x_{-}, x_{+}\right)$. Actually, we shall obtain a more general result.

Proposition 2.8. Let a function $w(x)$ be differentiable on the interval $\left(x_{-}, x_{+}\right)$except at a finite number of points. Suppose that $w(x)$ and $w^{\prime}(x)$ are locally bounded functions and that, for some $N$,

$$
|w(x)| \leq\left.\left. C q(x)\left|\int_{0}^{x}\right| q(y)\right|^{1 / 2} d y\right|^{N}
$$

if $|x|$ is large. Then under Assumptions 2.1 and 2.2 we have the asymptotic relation

$$
\int_{x_{1}}^{ \pm \infty} w(x) u_{ \pm}^{2}(x) d x=2^{-1} \pi \hbar^{1 / 3} \int_{x_{1}}^{x_{ \pm}} w(x)(\lambda-v(x))^{-1 / 2} d x+O\left(\hbar^{2 / 3}\right) .
$$

Proof. We prove (2.23) for the sign "+", omit this index and add $\hbar$. Using the asymptotic relations (2.14), (2.15) and the estimate $\xi^{\prime}(x) \geq c>0$, we see that

$$
\int_{x_{+}}^{x_{+}+1} w(x) u_{\hbar}^{2}(x) d x \leq C \int_{x_{+}}^{\infty} \xi^{\prime}(x) \operatorname{Ai}^{2}\left(\hbar^{-2 / 3} \xi(x)\right) d x=C_{1} \hbar^{2 / 3}
$$

Similarly, using identity (2.10) and condition (2.22), we find

$$
\int_{x_{+}+1}^{\infty} w(x) u_{\hbar}^{2}(x) d x \leq C \int_{x_{+}+1}^{\infty} \xi^{\prime}(x) \xi^{3 N / 2+1}(x) \operatorname{Ai}^{2}\left(\hbar^{-2 / 3} \xi(x)\right) d x=O\left(\hbar^{\infty}\right) .
$$

Suppose that $\delta_{\hbar} \rightarrow 0$ as $\hbar \rightarrow 0$, but $\delta_{\hbar} \hbar^{-2 / 3} \geq c>0$. The integral of $u_{\hbar}^{2}(x)$ over $\left(x_{+}-\delta_{\hbar}, x_{+}\right)$is estimated by $C \delta_{\hbar}$ because, by (2.20), the functions $u_{\hbar}(x)$ are uniformly bounded in a neighborhood of the point $x_{+}$. On the interval $\left(x_{1}, x_{+}-\delta_{\hbar}\right)$, we have

$$
\begin{aligned}
\int_{x_{1}}^{x_{+}-\delta_{\hbar}} w(x) u_{\hbar}^{2}(x) & d x=\pi \hbar^{1 / 3} \int_{x_{1}}^{x_{+}-\delta_{\hbar}} w(x)|q(x)|^{-1 / 2} \\
\times & \sin ^{2}\left(\hbar^{-1} \int_{x}^{x_{+}}|q(y)|^{1 / 2} d y+\pi / 4\right) d x+O\left(\hbar^{4 / 3} \delta_{\hbar}^{-1}\right) .
\end{aligned}
$$

Indeed, the asymptotic formula (2.19) shows that we need to check that the integrals

$$
\hbar^{7 / 3} \int_{x_{1}}^{x_{+}-\delta_{\hbar}}\left|x-x_{+}\right|^{-7 / 2} d x \quad \text { and } \quad \hbar^{4 / 3} \int_{x_{1}}^{x_{+}-\delta_{\hbar}}|q(x)|^{-1 / 4}\left|x-x_{+}\right|^{-7 / 4} d x
$$

are $O\left(\hbar^{4 / 3} \delta_{\hbar}^{-1}\right)$. The first of them equals $C \hbar^{7 / 3} \delta_{\hbar}^{-5 / 2}$, which is $O\left(\hbar^{4 / 3} \delta_{\hbar}^{-1}\right)$ because $\hbar=O\left(\delta_{\hbar}^{3 / 2}\right)$. To estimate the second integral, we observe additionally that

$$
|q(x)| \geq c\left(x_{+}-x\right), \quad c>0 .
$$

Next, we replace $\sin ^{2}(\cdot)$ on the right-hand side of (2.24) by $1 / 2$. Let us estimate the error. Integrating by parts separately on every interval where $w(x)$ is differentiable, we see that

$$
\begin{aligned}
\int_{x_{1}}^{x_{+}-\delta_{\hbar}} & w(x)|q(x)|^{-1 / 2} \exp \left(2 i \hbar^{-1} \int_{x}^{x_{+}}|q(y)|^{1 / 2} d y\right) d x \\
= & -2^{-1} i \hbar w\left(x_{+}-\delta_{\hbar}\right) q\left(x_{+}-\delta_{\hbar}\right)^{-1} \exp \left(2 i \hbar^{-1} \int_{x_{+}-\delta_{\hbar}}^{x_{+}}|q(y)|^{1 / 2} d y\right) \\
+ & 2^{-1} i \hbar \int_{x_{1}}^{x_{+}-\delta_{\hbar}}\left(w^{\prime}(x) q(x)^{-1}-v^{\prime}(x) q(x)^{-2} w(x)\right) \\
& \times \exp \left(2 i \hbar^{-1} \int_{x}^{x_{+}}|q(y)|^{1 / 2} d y\right) d x+O(\hbar) .
\end{aligned}
$$


Here the right-hand side is bounded by

$$
C \hbar\left(1+\left|q\left(x_{+}-\delta_{\hbar}\right)\right|^{-1}+\int_{x_{1}}^{x_{+}-\delta_{\hbar}} q(x)^{-2} d x\right),
$$

which does not exceed $C \hbar \delta_{\hbar}^{-1}$ by (2.25). Thus, from (2.24) it follows that

$$
\int_{x_{1}}^{x_{+}-\delta_{\hbar}} w(x) u_{\hbar}^{2}(x) d x=2^{-1} \pi \hbar^{1 / 3} \int_{x_{1}}^{x_{+}-\delta_{\hbar}} w(x)(\lambda-v(x))^{-1 / 2} d x+O\left(\hbar^{4 / 3} \delta_{\hbar}^{-1}\right) .
$$

Finally, making an error of order $O\left(\hbar^{1 / 3} \delta_{\hbar}^{1 / 2}\right)$, we can extend the integral on the righthand side to the entire interval $\left(x_{1}, x_{+}\right)$. Setting $\delta_{\hbar}=\hbar^{2 / 3}$ and collecting the results obtained, we arrive at the asymptotic relation (2.23).

Of course, (2.22) is a very mild restriction. It is satisfied for $v(x)=w(x)$. It is also fulfilled for all functions $v(x)$ if $w(x)$ is bounded by some power of $|x|$ at infinity and is even less restrictive if $v(x) \rightarrow \infty$ as $|x| \rightarrow \infty$. In particular, setting $w(x)=1$, we obtain the following statement.

Corollary 2.9. We have

$$
\int_{x_{1}}^{ \pm \infty} u_{ \pm}^{2}(x) d x=2^{-1} \pi \hbar^{1 / 3} \int_{x_{1}}^{x_{ \pm}}(\lambda-v(x))^{-1 / 2} d x+O\left(\hbar^{2 / 3}\right) .
$$

\section{§3. Proof of Theorem 2.5}

3.1. We shall prove Theorem 2.5 for the sign "+" and omit this index. On the contrary, we add the index $\hbar$ to emphasize the dependence on it of various objects. Let $x_{1} \in$ $\left(x_{-}, x_{+}\right), x \in\left(x_{1}, \infty\right)$, and let the function $\xi(x)$ be defined by formulas (2.8). By Lemma 2.3. we have $\xi(x) \in\left(\xi_{1}, \infty\right)$, where $\xi_{1}=\xi\left(x_{1}\right)$ and $x$ can be viewed as a function of $\xi$ if $\xi \in\left(\xi_{1}, \infty\right)$.

We make the change of variables $x \mapsto \xi$ in equation (2.1) and set

$$
u_{\hbar}(x)=\xi^{\prime}(x)^{-1 / 2} f_{\hbar}\left(\hbar^{-2 / 3} \xi(x)\right) .
$$

Then, using identity (2.10), we obtain

$$
-f_{\hbar}^{\prime \prime}\left(\hbar^{-2 / 3} \xi\right)+\hbar^{-2 / 3} \xi f_{\hbar}\left(\hbar^{-2 / 3} \xi\right)=\hbar^{4 / 3} r(\xi) f_{\hbar}\left(\hbar^{-2 / 3} \xi\right),
$$

where

$$
r(\xi)=p(x(\xi))
$$

and $p(x)$ is defined by formula (2.11). By (2.13), we have the estimate

$$
|r(\xi)| \leq C(1+|\xi|)^{-1 / 2-\rho}, \quad \rho=3 \min \left\{\rho_{0}-1,1\right\} / 2>0 .
$$

Setting $t=\hbar^{-2 / 3} \xi$ in (3.2), we get the following intermediary result.

Lemma 3.1. Let $t=\hbar^{-2 / 3} \xi(x)$, and let the functions $u_{\hbar}(x)$ and $f_{\hbar}(t)$ be related by formula (3.1). Then equation (2.1) for $x \geq x_{1}$ is equivalent to the equation

$$
-f_{\hbar}^{\prime \prime}(t)+t f_{\hbar}(t)=R_{\hbar}(t) f_{\hbar}(t) \quad \text { for } \quad t \geq \xi_{1} \hbar^{-2 / 3},
$$

where

$$
R_{\hbar}(t)=\hbar^{4 / 3} r\left(\hbar^{2 / 3} t\right) .
$$


3.2. We reduce the differential equation (3.5) to a Volterra integral equation. Set

$$
K_{\hbar}(t, s)=-\pi(\operatorname{Ai}(t) \operatorname{Bi}(s)-\operatorname{Ai}(s) \operatorname{Bi}(t)) R_{\hbar}(s), \quad s \geq t,
$$

and consider the equation

$$
f_{\hbar}(t)=\operatorname{Ai}(t)+\int_{t}^{\infty} K_{\hbar}(t, s) f_{\hbar}(s) d s .
$$

Differentiating it twice, we see that its solution also satisfies (3.5). We shall study equations (3.5) or (3.8) separately for $t \geq 0$ and $t \leq 0$.

Lemma 3.2. For $t \geq 0$, equation (3.5) has a solution $f_{\hbar}(t)$ such that

$$
f_{\hbar}(t)=\operatorname{Ai}(t)\left(1+\eta_{\hbar}(t)\right)
$$

where

$$
\left|\eta_{\hbar}(t)\right| \leq C \hbar\left(1+\hbar^{2 / 3} t\right)^{-\rho}, \quad \rho=3 \min \left\{\rho_{0}-1,1\right\} / 2>0 .
$$

Proof. Making the multiplicative change of variables

$$
f_{\hbar}(t)=\operatorname{Ai}(t) g_{\hbar}(t)
$$

and using (2.7), we rewrite equation (3.8) as

$$
g_{\hbar}(t)=1-\int_{t}^{\infty} L_{\hbar}(t, s) g_{\hbar}(s) d s,
$$

where

$$
L_{\hbar}(t, s)=\operatorname{Ai}(t)^{-1} K_{\hbar}(t, s) \operatorname{Ai}(s)=\int_{t}^{s} \operatorname{Ai}^{-2}(\tau) d \tau \operatorname{Ai}^{2}(s) R_{\hbar}(s), \quad s \geq t .
$$

Relation (2.3) implies that

$$
\int_{t}^{s} \mathrm{Ai}^{-2}(\tau) d \tau \leq C \exp \left(4 s^{3 / 2} / 3\right)
$$

so that, by (3.4) and (3.6),

$$
\left|L_{\hbar}(t, s)\right| \leq C \hbar^{4 / 3} s^{-1 / 2}\left(1+\hbar^{2 / 3} s\right)^{-1 / 2-\rho}, \quad 0 \leq t \leq s .
$$

This estimate allows us to solve equation (3.12) by iterations. In particular, the solution of (3.12) satisfies the estimate

$$
\left|g_{\hbar}(t)-1\right| \leq C \int_{t}^{\infty}\left|L_{\hbar}(t, s)\right| d s .
$$

Now estimate (3.10) on the remainder $\eta_{\hbar}(t)=g_{\hbar}(t)-1$ follows again from (3.13).

Putting formulas (3.1) and (3.9) together, we obtain representation (2.14) with $\varepsilon_{\hbar}(x)=$ $\eta_{\hbar}\left(\hbar^{-2 / 3} \xi(x)\right)$. Estimate (3.10) implies (2.15). This leads to the assertion of Theorem 2.5 for $x \geq x_{+}$. In particular, for a fixed $\hbar$, we get Proposition 2.4.

Next, we consider the case where $t \leq 0$.

Lemma 3.3. For $t \in\left[\hbar^{-2 / 3} \xi_{1}, 0\right]$, the solution $f_{\hbar}(t)$ of equation (3.5) satisfies the estimate

$$
\left|f_{\hbar}(t)-\operatorname{Ai}(t)\right| \leq C \hbar(1+|t|)^{-1 / 4}
$$


Proof. We rewrite equation (3.8) as

$$
f_{\hbar}(t)=f_{\hbar}^{(0)}(t)+\int_{t}^{0} K_{\hbar}(t, s) f_{\hbar}(s) d s,
$$

with the new "free" term

$$
f_{\hbar}^{(0)}(t)=\operatorname{Ai}(t)+f_{\hbar}^{(1)}(t), \quad f_{\hbar}^{(1)}(t)=\int_{0}^{\infty} K_{\hbar}(t, s) f_{\hbar}(s) d s .
$$

By (2.3) and (2.6), we have

$$
\mathrm{Ai}^{2}(t)+\mathrm{Ai}(t) \mathrm{Bi}(t) \leq C(1+t)^{-1 / 2}, \quad t \geq 0,
$$

and relations (2.4) and (2.5) show that

$$
|\mathrm{Ai}(t)|+|\operatorname{Bi}(t)| \leq C(1+|t|)^{-1 / 4}, \quad t \leq 0 .
$$

Therefore, using (3.4), (3.6), and (3.7), we find that

$$
\begin{aligned}
\left|f_{\hbar}^{(1)}(t)\right| & \leq C\left(|\mathrm{Ai}(t)| \int_{0}^{\infty} \operatorname{Ai}(s) \operatorname{Bi}(s)\left|R_{\hbar}(s)\right| d s+|\operatorname{Bi}(t)| \int_{0}^{\infty} \operatorname{Ai}^{2}(s)\left|R_{\hbar}(s)\right| d s\right) \\
& \leq C_{1}(1+|t|)^{-1 / 4} \hbar^{4 / 3} \int_{0}^{\infty} s^{-1 / 2}\left(1+\hbar^{2 / 3} s\right)^{-1 / 2-\rho} d s \\
& \leq C_{2}(1+|t|)^{-1 / 4} \hbar
\end{aligned}
$$

Now we consider equation (3.15). Estimates (3.4) and (3.17) imply that its kernel satisfies the bound

$$
\left|K_{\hbar}(t, s)\right| \leq C \hbar^{4 / 3}(1+|t|)^{-1 / 4}(1+|s|)^{-1 / 4} r\left(\hbar^{2 / 3} s\right), \quad t \leq s \leq 0,
$$

where the function $r\left(\hbar^{2 / 3} s\right)$ can be estimated by a constant. Thus, solving (3.15) again by iterations, we obtain the estimate

$$
\begin{aligned}
\left|f_{\hbar}(t)-f_{\hbar}^{(0)}(t)\right| & \leq C_{1} \int_{t}^{0}\left|K_{\hbar}(t, s)\right|(1+|s|)^{-1 / 4} d s \\
& \leq C_{2} \hbar^{4 / 3} \int_{t}^{0}(1+|s|)^{-3 / 4} d s \leq C_{3} \hbar^{4 / 3}(1+|t|)^{1 / 4} .
\end{aligned}
$$

If $t \in\left[\hbar^{-2 / 3} \xi_{1}, 0\right]$, then, to get estimate (3.14), it suffices to combine (3.16) with estimates (3.18) and (3.20).

In view of formula (3.1), this lemma yields the result of Theorem 2.5 for $x \in\left[x_{1}, x_{+}\right]$.

Differentiating the integral equation (3.8) with respect to $t$, we obtain asymptotic relations for $f_{\hbar}^{\prime}(t)$ and then for $u_{\hbar}^{\prime}(x)$. This concludes the proof of Theorem 2.5 .

\section{§4. Semiclassical asymptotic expressions for eigenfunctions}

4.1. Let $\lambda=\lambda(\hbar)$ be an eigenvalue of the Schrödinger operator $H_{\hbar}=-\hbar^{2} d^{2} / d x^{2}+v(x)$ in a neighborhood of a noncritical point $\lambda_{0}$. Then the solutions $u_{ \pm}(x)$ are proportional:

$$
u_{-}(x ; \lambda, \hbar)=a(\lambda, \hbar) u_{+}(x ; \lambda, \hbar) .
$$

Choose an arbitrary interior point $x$ of the interval $\left(x_{-}(\lambda), x_{+}(\lambda)\right)$. To calculate the Wronskian of $u_{+}(x)$ and $u_{-}(x)$, we use the asymptotic relations (2.19) and (2.21). Setting

$$
\varphi_{ \pm}(x ; \lambda)= \pm \int_{x}^{x_{ \pm}(\lambda)}(\lambda-v(y))^{1 / 2} d y, \quad x \in\left(x_{-}(\lambda), x_{+}(\lambda)\right),
$$


we find that

$$
\begin{aligned}
w(\lambda, \hbar)= & u_{+}(x ; \lambda, \hbar) u_{-}^{\prime}(x ; \lambda, \hbar)-u_{-}(x ; \lambda, \hbar) u_{+}^{\prime}(x ; \lambda, \hbar) \\
= & \pi \hbar^{-2 / 3}\left(\sin \left(\hbar^{-1} \varphi_{+}(x ; \lambda)+\pi / 4\right) \cos \left(\hbar^{-1} \varphi_{-}(x ; \lambda)+\pi / 4\right)\right. \\
& \quad+\cos \left(\hbar^{-1} \varphi_{+}(x ; \lambda)+\pi / 4\right) \sin \left(\hbar^{-1} \varphi_{-}(x ; \lambda+\pi / 4)\right)+O\left(\hbar^{1 / 3}\right) \\
= & \pi \hbar^{-2 / 3} \sin \left(\hbar^{-1} \Phi(\lambda)+\pi / 2\right)+O\left(\hbar^{1 / 3}\right),
\end{aligned}
$$

where $\Phi(\lambda)=\varphi_{+}(x ; \lambda)+\varphi_{-}(x ; \lambda)$, so that

$$
\Phi(\lambda)=\int_{x_{-}(\lambda)}^{x_{+}(\lambda)}(\lambda-v(y))^{1 / 2} d y .
$$

Since $w(\lambda, \hbar)=0$, we see that

$$
\sin \left(\hbar^{-1} \Phi(\lambda)+\pi / 2\right)=O(\hbar),
$$

whence

$$
\int_{x_{-}(\lambda)}^{x_{+}(\lambda)}(\lambda-v(x))^{1 / 2} d x=\pi(n+1 / 2) \hbar+O\left(\hbar^{2}\right)
$$

for some integer $n=n(\lambda, \hbar)$. This gives us the famous Bohr-Sommerfeld quantization condition.

Now, suppose that a number of the form $\pi(n+1 / 2) \hbar$ belongs to a neighborhood of $\lambda_{0}$. We check that there exists an eigenvalue $\lambda_{n}(\hbar)$ of the operator $H_{\hbar}$ satisfying the estimate

$$
\left|\Phi\left(\lambda_{n}(\hbar)\right)-\pi(n+1 / 2) \hbar\right| \leq C \hbar^{2} .
$$

Since $u_{ \pm} \in L^{2}\left(\mathbb{R}_{ \pm}\right)$, it suffices to show that $w(\lambda, \hbar)=0$ for some $\lambda=\lambda_{n}(\hbar)$ satisfying (4.6). Using the equation $\lambda-v\left(x_{ \pm}(\lambda)\right)=0$, we see that

$$
\Phi^{\prime}(\lambda)=2^{-1} \int_{x_{-}(\lambda)}^{x_{+}(\lambda)}(\lambda-v(y))^{-1 / 2} d y>0 .
$$

Hence, $\Phi$ is a one-to-one mapping of a neighborhood of $\lambda_{0}$ onto a neighborhood of $\mu_{0}=\Phi\left(\lambda_{0}\right)$. Set $\mu=\Phi(\lambda)$ and

$$
\epsilon(\mu, \hbar)=\pi^{-1} \hbar^{2 / 3} w\left(\Phi^{-1}(\mu), \hbar\right)-\sin \left(\hbar^{-1} \mu+\pi / 2\right) .
$$

By (4.3), this function satisfies $|\epsilon(\mu, \hbar)| \leq C \hbar$ with a constant $C$ independent of $\hbar$ and $\mu$ in a neighborhood of $\mu_{0}$. We need to show that the equation

$$
\sin \left(\hbar^{-1} \mu+\pi / 2\right)+\epsilon(\mu, \hbar)=0
$$

has a solution $\mu_{n}(\hbar)$ obeying the estimate

$$
\left|\mu_{n}(\hbar)-\pi(n+1 / 2) \hbar\right| \leq C \hbar^{2} .
$$

Setting $s=\hbar^{-1} \mu+\pi / 2$, we see that this assertion is equivalent to the existence of a solution $s=s_{n}(\hbar)$ of the equation

$$
\sin s+\epsilon(\hbar(s-\pi / 2), \hbar)=0
$$

obeying the estimate

$$
\left|s_{n}(\hbar)-\pi(n+1)\right| \leq C \hbar .
$$

The last fact is obvious because $\epsilon(\hbar(s-\pi / 2), \hbar)=O(\hbar)$. 
Next, we show that for every $n$ there is only one eigenvalue of the operator $H_{\hbar}$ satisfying (4.6). For that, we must check that equation (4.9) cannot have two solutions satisfying (4.10). Supposing the contrary, we find a point $\widetilde{s}=\widetilde{s}_{n}(\hbar)$ such that

$$
\cos \widetilde{s}=-\hbar \frac{\partial \epsilon}{\partial \mu}(\hbar(\widetilde{s}-\pi / 2), \hbar)
$$

and $\widetilde{s}_{n}(\hbar)=\pi(n+1)+O(\hbar)$. Observe that relation (4.3) can be differentiated in $\lambda$, which yields

$$
\partial w(\lambda, \hbar) / d \lambda=\pi \Phi^{\prime}(\lambda) \hbar^{-5 / 3} \cos \left(\hbar^{-1} \Phi(\lambda)+\pi / 2\right)+O\left(\hbar^{-2 / 3}\right) .
$$

It follows that the function (4.8) obeys $\partial \varepsilon(\mu, \hbar) / d \mu=O(1)$. Thus, the right-hand side of equation (4.11) is $O(\hbar)$, while its left-hand side tends to $(-1)^{n+1}$ as $\hbar \rightarrow 0$.

Finally, plugging the asymptotic formulas (2.19) and (2.21) into (4.1), we find that

$$
\begin{aligned}
& \sin \left(\hbar^{-1} \varphi_{-}(x ; \lambda)+\pi / 4\right)+O(\hbar) \\
& \quad=a(\lambda, \hbar)\left(\sin \left(\hbar^{-1} \varphi_{+}(x ; \lambda)+\pi / 4\right)+O(\hbar)\right)
\end{aligned}
$$

and

$$
\begin{aligned}
& \cos \left(\hbar^{-1} \varphi_{-}(x ; \lambda)+\pi / 4\right)+O(\hbar) \\
& \quad=-a(\lambda, \hbar)\left(\cos \left(\hbar^{-1} \varphi_{+}(x ; \lambda)+\pi / 4\right)+O(\hbar)\right) .
\end{aligned}
$$

Together, these two relations imply that $|a(\lambda, \hbar)|=1+O(\hbar)$. Moreover, since

$$
\varphi_{+}(x ; \lambda)+\varphi_{-}(x ; \lambda)=\pi(n+1 / 2) \hbar+O\left(\hbar^{2}\right),
$$

from (4.12) and (4.13) it follows that

$$
a(\lambda, \hbar)=(-1)^{n}+O(\hbar) .
$$

Thus, we have obtained the following result.

Theorem 4.1. Let Assumptions 2.1 and 2.2 hold true for a point $\lambda_{0}$. Suppose that an eigenvalue $\lambda=\lambda(\hbar)$ of the operator $H_{\hbar}$ belongs to a neighborhood of $\lambda_{0}$. Then, necessarily, condition (4.5) is satisfied with some integer $n=n(\lambda, \hbar)$. Conversely, for every $n$ such that $\pi(n+1 / 2) \hbar$ belongs to a neighborhood of $\Phi\left(\lambda_{0}\right)$, there exists an eigenvalue $\lambda_{n}(\hbar)$ of the operator $H_{\hbar}$ satisfying estimate (4.6) with a constant $C$ not depending on $n$ and $\hbar$. Such an eigenvalue $\lambda_{n}(\hbar)$ is unique. Moreover, the coefficient $a(\lambda, \hbar)$ in (4.1) has the asymptotic formula (4.14), where $n$ is the same as in (4.5).

Corollary 4.2. Let an interval $\left(a_{1}, a_{2}\right)$ belong to a neighborhood of a point $\lambda_{0}$ satisfying Assumptions 2.1] and 2.2. Then the total number $N_{\hbar}$ of eigenvalues of the operator $H_{\hbar}$ in this interval equals

$$
N_{\hbar}=\pi^{-1}\left(\Phi\left(a_{2}\right)-\Phi\left(a_{1}\right)\right) \hbar^{-1}+\epsilon(\hbar),
$$

where $|\epsilon(\hbar)| \leq 1$ for sufficiently small $\hbar$.

Proof. By Theorem 4.1, there is exactly one eigenvalue of the operator $H_{\hbar}$ in a neighborhood of size $C \hbar^{2}$ of every point $\Phi^{-1}(\pi(n+1 / 2) \hbar)$. These neighborhoods have empty mutual intersections for sufficiently small $\hbar$. Thus, $N_{\hbar}$ equals the number of points $\pi(n+1 / 2) \hbar$ lying in the interval $\left(\Phi\left(a_{1}\right), \Phi\left(a_{2}\right)\right)$. Clearly, this number equals the righthand side of (4.15).

Remark 4.3. Suppose that Assumptions 2.1 and 2.2 hold true for all $\lambda \in\left[a_{1}, a_{2}\right]$. Then the remainders in the asymptotic formulas of this paper can be estimated uniformly in $\lambda \in\left[a_{1}, a_{2}\right]$. Formula (4.15) also remains true for such $\left(a_{1}, a_{2}\right)$. 
Note that (4.4) can be rewritten as

$$
\Phi(\lambda)=2^{-1} \iint_{p^{2}+v(x) \leq \lambda} d p d x
$$

Indeed, integrating on the right-hand side first over $p$, we obtain the right-hand side of (4.4). It follows that the asymptotic coefficient in (4.15) is the volume of a part of the phase space:

$$
\Phi\left(a_{2}\right)-\Phi\left(a_{1}\right)=2^{-1} \text { meas }\left\{(x, p) \in \mathbb{R}^{2}: a_{1} \leq p^{2}+v(x) \leq a_{2}\right\} .
$$

Thus, relation (4.15) is the semiclassical Weyl formula with a strong estimate of the remainder.

4.2. Let $\psi(x)=\psi(x ; \lambda, \hbar)$ denote the eigenfunction of the operator $H_{\hbar}$ corresponding to its eigenvalue $\lambda$. We suppose that $\psi=\bar{\psi} \in L^{2}\left(\mathbb{R}_{+}\right)$and $\|\psi\|=1$, which fixes $\psi$ up to the sign. Clearly,

$$
\psi(x)=c_{ \pm} u_{ \pm}(x), \quad c_{ \pm}=c_{ \pm}(\lambda, \hbar)
$$

where, by (4.14),

$$
\left|c_{+}(\lambda, \hbar)\right|=\left|c_{-}(\lambda, \hbar)\right|(1+O(\hbar))
$$

Therefore, Corollary 2.9 shows that

$$
\left|c_{ \pm}(\lambda, \hbar)\right|=2^{1 / 2} \pi^{-1 / 2} \hbar^{-1 / 6}\left(\int_{x_{-}(\lambda)}^{x_{+}(\lambda)}(\lambda-v(x))^{-1 / 2} d x\right)^{-1 / 2}+O\left(\hbar^{1 / 6}\right),
$$

and an application of Theorem 2.5 yields the following result.

Theorem 4.4. Under the assumptions of Theorem 4.1, let $\psi(\lambda, \hbar)$ denote the real normalized eigenfunction (defined up to a sign) of the operator $H_{\hbar}$ corresponding to its eigenvalue $\lambda=\lambda(\hbar)$. Let $x_{1}$ be an arbitrary point in the interval $\left(x_{-}(\lambda), x_{+}(\lambda)\right)$. Then, for $x \in\left(x_{1}, \infty\right)$, the asymptotic behavior of $\psi(x ; \lambda, \hbar)$ as $\hbar \rightarrow 0$ is given by formulas (4.17), (4.18) for the sign " + " and the asymptotic relations of Theorem 2.5 for the function $u_{+}(x ; \lambda, \hbar)$. Similarly, for $x \in\left(-\infty, x_{1}\right)$, the asymptotic behavior of $\psi(x ; \lambda, \hbar)$ as $\hbar \rightarrow 0$ is given by formulas (4.17), (4.18) for the sign " - " and the asymptotic relations of Theorem 2.5 for the function $u_{-}(x ; \lambda, \hbar)$. In neighborhoods of the turning points, the estimate

$$
|\psi(x ; \lambda, \hbar)| \leq C\left(\hbar^{2 / 3}+\left|x-x_{ \pm}\right|\right)^{-1 / 4}
$$

is valid with a constant $C$ independent of $\hbar$.

Recalling formula (2.23), we see that this result can be supplemented by the following statement.

Proposition 4.5. Let a function $w$ satisfy the assumptions of Proposition 2.8, Then under the assumptions of Theorem 4.4 we have

$$
\begin{aligned}
\int_{-\infty}^{\infty} w(x) \psi^{2}(x ; \lambda, \hbar) d x= & \int_{x_{-}(\lambda)}^{x_{+}(\lambda)} w(x)(\lambda-v(x))^{-1 / 2} d x \\
& \times\left(\int_{x_{-}(\lambda)}^{x_{+}(\lambda)}(\lambda-v(x))^{-1 / 2} d x\right)^{-1}+O\left(\hbar^{1 / 3}\right) .
\end{aligned}
$$

In particular, this relation applies to the potential energy

$$
V(\lambda, \hbar)=\int_{-\infty}^{\infty} v(x) \psi^{2}(x ; \lambda, \hbar) d x
$$

and, due to the energy conservation $K(\lambda, \hbar)+V(\lambda, \hbar)=\lambda$, we also obtain the asymptotic expansion of the kinetic energy. 
Corollary 4.6. Under the assumptions of Theorem 4.4, the asymptotic relation (1.3) holds true with the leading term $K_{\mathrm{cl}}(\lambda)$ given by (1.4).

Since $K_{\mathrm{cl}}(\lambda)>0$, for small $\hbar$ the kinetic energy satisfies $K(\lambda, \hbar) \geq c>0$, or equivalently, for the potential energy we have $V(\lambda, \hbar) \leq \lambda-c$. This implies that the eigenfunctions $\psi(x ; \lambda, \hbar)$ are not too strongly localized in neighborhoods of the turning points $x_{ \pm}(\lambda)$. Estimate (4.19) allows us to refine this statement.

Proposition 4.7. Under the assumptions of Theorem 4.1, let $\|\psi(\lambda, \hbar)\|=1$. Then

$$
\int_{x_{ \pm}(\lambda)-\delta}^{x_{ \pm}(\lambda)+\delta} \psi^{2}(x ; \lambda, \hbar) d x \leq C \delta^{1 / 2}
$$

where the constant $C$ does not depend on $\hbar$.

Remark 4.8. The asymptotic formulas (2.14) and (4.18) imply that

$$
\left|\psi\left(x_{ \pm}(\lambda) ; \lambda, \hbar\right)\right|=\alpha_{ \pm}(\lambda) \hbar^{-1 / 6}\left(1+O\left(\hbar^{1 / 3}\right)\right)
$$

where

$$
\alpha_{ \pm}(\lambda)=2^{1 / 2} \pi^{-1 / 2}\left(\int_{x_{-}(\lambda)}^{x_{+}(\lambda)}(\lambda-v(x))^{-1 / 2} d x\right)^{-1 / 2}\left|v^{\prime}\left(x_{ \pm}(\lambda)\right)\right|^{-1 / 6} \operatorname{Ai}(0) \neq 0 .
$$

This contradicts the claim of Theorem 7.1 of $[8$ saying that the normalized eigenfunctions are uniformly bounded in neighborhoods of turning points.

4.3. Recall that a classical particle (of mass $m$ and energy $\lambda$ ) moves periodically (see, e.g., [6]) in a potential well bounded by the points $x_{-}=x_{-}(\lambda)$ and $x_{+}=x_{+}(\lambda)$ such that $v\left(x_{ \pm}\right)=\lambda$. Let us check that the asymptotic coefficient $K_{\mathrm{cl}}$ in (1.3) coincides with the averaged (over the period $T=T(\lambda)$ ) value

$$
K_{\mathrm{av}}=T^{-1} \int_{0}^{T} K(t) d t
$$

of the classical kinetic energy

$$
K(t)=m x^{\prime}(t)^{2} / 2=\lambda-v(x(t)) .
$$

Since

$$
d t=x^{\prime}(t)^{-1} d x=(m / 2)^{1 / 2}(\lambda-v(x))^{-1 / 2} d x,
$$

the period is given by the formula

$$
T=2 \int_{x_{-}}^{x_{+}} \frac{d t}{d x} d x=(2 m)^{1 / 2} \int_{x_{-}}^{x_{+}}(\lambda-v(x))^{-1 / 2} d x,
$$

and

$$
K_{\mathrm{av}}=T^{-1} \int_{0}^{T}(\lambda-v(x(t))) d t=2(m / 2)^{1 / 2} T^{-1} \int_{x_{-}}^{x_{+}}(\lambda-v(x))^{1 / 2} d x .
$$

Putting these two relations together, we obtain the same expression (1.4) for $K_{\mathrm{av}}$ as for $K_{\mathrm{cl}}$. This proves the identity

$$
K_{\mathrm{cl}}(\lambda)=K_{\mathrm{av}}(\lambda)
$$

Also, we note that

$$
K_{\mathrm{cl}}(\lambda)=(2 d \ln \Phi(\lambda) / d \lambda)^{-1},
$$

where the function $\Phi(\lambda)$ is defined by formulas (4.4), or equivalently, (4.16). For the proof, it suffices to plug the representation (4.7) for the function $\Phi^{\prime}(\lambda)$ into formula (1.4). 


\section{$\S 5$. Discontinuous potentials}

5.1. Away from the turning points, the assumptions on $v(x)$ can be relaxed somewhat. Consider, for example, an interval $\left(x_{-}+\delta, x_{+}-\delta\right)$, where $\delta>0$. There, it suffices to require that $v \in C^{1}$ and that $v^{\prime}$ be absolutely continuous, so that $v^{\prime \prime} \in L^{1}$ (instead of $v \in C^{2}$ ). In this case the function $r(\xi)$ defined by formulas (2.11) and (3.3) only belongs to $L^{1}$, so that the factor $r\left(\hbar^{2 / 3} s\right)$ on the right-hand side of (3.19) cannot be neglected. Therefore (cf. (3.20)), we have the estimate

$$
\begin{aligned}
\int_{t}^{0}\left|K_{\hbar}(t, s)\right|(1+|s|)^{-1 / 4} d s & \leq C \hbar^{4 / 3}(1+|t|)^{-1 / 4} \int_{t}^{0}\left|r\left(\hbar^{2 / 3} s\right)\right| d s \\
& \leq C_{1} \hbar^{2 / 3}(1+|t|)^{-1 / 4} \int_{\xi\left(x_{-}+\delta\right)}^{0}|r(s)| d s .
\end{aligned}
$$

It follows that instead of (3.14) we have a slightly weaker estimate with $\hbar^{2 / 3}$ in place of $\hbar$ on the right-hand side. All other estimates remain unchanged. Thus, Theorem 2.5] is true with slightly weaker estimates of the remainders in asymptotic formulas for $u_{ \pm}(x ; \lambda, \hbar)$ inside the interval $\left(x_{-}+\delta, x_{+}-\delta\right)$. Repeating the arguments of $\S 4$, we get the following result.

Proposition 5.1. Under the assumptions above, all results of Theorem 4.1 (and of Corollary 4.2) about eigenvalues of the operators $H_{\hbar}$ remain true with the remainders $O\left(\hbar^{5 / 3}\right)$ in (4.5), $O\left(\hbar^{2 / 3}\right)$ in (4.14), and $C \hbar^{5 / 3}$ on the right-hand side of (4.6). Theorem 4.4 about the corresponding eigenfunctions remains true with the remainders $O\left(\hbar^{5 / 6}\left|x-x_{ \pm}\right|^{-7 / 4}\right)$ in (2.19), $O\left(\hbar^{-1 / 6}\left|x-x_{ \pm}\right|^{-7 / 4}\right)$ in (2.21), and $O\left(\hbar^{1 / 18}\right)$ in (4.18).

5.2. Our goal in this subsection is to extend the results of $\S 4$ to functions $v(x)$ with a singular point $x_{0}$ inside a potential well.

We suppose that Assumption 2.1 is fulfilled everywhere except at a point $x_{0}$ and that Assumption 2.2 is fulfilled for some $\lambda_{0}$ such that $x_{0}$ is an interior point of the interval $\left(x_{-}\left(\lambda_{0}\right), x_{+}\left(\lambda_{0}\right)\right)$. Assume that $v(x)$ has finite limits at $x_{0}$, but the left and the right limits might be different. Finally, we require that $v^{\prime} \in L^{2}\left(x_{0}, x_{0} \pm \delta\right)$ and $v^{\prime \prime} \in L^{1}\left(x_{0}, x_{0} \pm \delta\right)$ for some $\delta>0$.

Now we can construct solutions $u_{+}(x)$ and $u_{-}(x)$ of equation (1.1) on the intervals $\left(x_{0}, \infty\right)$ and $\left(-\infty, x_{0}\right)$, respectively. As usual, we define the function $r(\xi)$ by formulas (2.11) and (3.3). Since $r \in L^{1}\left(x_{0}, x_{0} \pm \delta\right)$, the limits $u_{ \pm}\left(x_{0} \pm 0\right)$ and $u_{ \pm}^{\prime}\left(x_{0} \pm 0\right)$ exist, and we can use the formulas (2.19) and (2.21) for these limits (with slightly weaker estimates of the remainders - see Subsection 5.1). It follows that the Wronskian $w(\lambda, \hbar)$ of $u_{+}$ and $u_{-}$calculated at the point $x_{0}$ is given by the expression (cf. (4.3) )

$$
\begin{aligned}
\pi \hbar^{-2 / 3} & \left(p\left(x_{0}, \lambda\right) \sin \left(\hbar^{-1} \varphi_{+}\left(x_{0} ; \lambda\right)+\pi / 4\right) \cos \left(\hbar^{-1} \varphi_{-}\left(x_{0} ; \lambda\right)+\pi / 4\right)\right. \\
& \left.+p\left(x_{0}, \lambda\right)^{-1} \cos \left(\hbar^{-1} \varphi_{+}\left(x_{0} ; \lambda\right)+\pi / 4\right) \sin \left(\hbar^{-1} \varphi_{-}\left(x_{0} ; \lambda\right)+\pi / 4\right)\right)+O(1)
\end{aligned}
$$

where

$$
p\left(x_{0}, \lambda\right)=\left(\lambda-v\left(x_{0}-0\right)\right)^{1 / 4}\left(\lambda-v\left(x_{0}+0\right)\right)^{-1 / 4} .
$$

Let an eigenvalue $\lambda$ of the operator $H_{\hbar}$ be close to $\lambda_{0}$. Since $w(\lambda, \hbar)=0$, we see that

$$
\begin{aligned}
& p\left(x_{0}, \lambda\right) \sin \left(\hbar^{-1} \varphi_{+}\left(x_{0} ; \lambda\right)+\pi / 4\right) \cos \left(\hbar^{-1} \varphi_{-}\left(x_{0} ; \lambda\right)+\pi / 4\right) \\
& \quad+p\left(x_{0}, \lambda\right)^{-1} \cos \left(\hbar^{-1} \varphi_{+}\left(x_{0} ; \lambda\right)+\pi / 4\right) \sin \left(\hbar^{-1} \varphi_{-}\left(x_{0} ; \lambda\right)+\pi / 4\right)=O\left(\hbar^{2 / 3}\right) .
\end{aligned}
$$

Formula (5.2) yields a generalization of the Bohr-Sommerfeld quantization condition (4.5) and reduces to it if $v\left(x_{0}+0\right)=v\left(x_{0}-0\right)$. 
Let the coefficient $a(\lambda, \hbar)$ be defined by (4.1). To calculate $|a(\lambda, \hbar)|$, we use relations (4.12) and (4.13) once again. However, now the additional factors $\left|q\left(x_{0}-0\right)\right|^{-1 / 4}$ and $\left|q\left(x_{0}-0\right)\right|^{1 / 4}$ appear on the left-hand sides. Similarly, the additional factors $\left|q\left(x_{0}+0\right)\right|^{-1 / 4}$ and $\left|q\left(x_{0}+0\right)\right|^{1 / 4}$ appear on the right-hand sides. This implies that

$$
\begin{aligned}
a^{2}(\lambda, \hbar)= & p^{2}\left(x_{0}, \lambda\right) \cos ^{2}\left(\hbar^{-1} \varphi_{-}\left(x_{0} ; \lambda\right)+\pi / 4\right) \\
& \quad+p^{-2}\left(x_{0}, \lambda\right) \sin ^{2}\left(\hbar^{-1} \varphi_{-}\left(x_{0} ; \lambda\right)+\pi / 4\right)+O\left(\hbar^{2 / 3}\right) \\
= & \left(p^{2}\left(x_{0}, \lambda\right) \sin ^{2}\left(\hbar^{-1} \varphi_{+}\left(x_{0} ; \lambda\right)+\pi / 4\right)\right. \\
\quad & \left.\quad p^{-2}\left(x_{0}, \lambda\right) \cos ^{2}\left(\hbar^{-1} \varphi_{+}\left(x_{0} ; \lambda\right)+\pi / 4\right)\right)^{-1}+O\left(\hbar^{2 / 3}\right) .
\end{aligned}
$$

As before, using formula (2.26) (where $x_{1}=x_{0}$ ) and the normalization condition $\|\psi\|=1$, we obtain explicit expressions for the absolute values of the constants $c_{ \pm}(\lambda, \hbar)$ in (4.17):

$$
\begin{aligned}
\left|c_{+}(\lambda, \hbar)\right|=2^{1 / 2} \pi^{-1 / 2} \hbar^{-1 / 6}( & \int_{x_{0}}^{x_{+}(\lambda)}(\lambda-v(x))^{-1 / 2} d x \\
& \left.+a^{-2}(\lambda, \hbar) \int_{x_{-}(\lambda)}^{x_{0}}(\lambda-v(x))^{-1 / 2} d x\right)^{-1 / 2}+O\left(\hbar^{1 / 18}\right)
\end{aligned}
$$

and

$$
\begin{aligned}
\left|c_{-}(\lambda, \hbar)\right|=2^{1 / 2} \pi^{-1 / 2} \hbar^{-1 / 6}( & a^{2}(\lambda, \hbar) \int_{x_{0}}^{x_{+}(\lambda)}(\lambda-v(x))^{-1 / 2} d x \\
& \left.+\int_{x_{-}(\lambda)}^{x_{0}}(\lambda-v(x))^{-1 / 2} d x\right)^{-1 / 2}+O\left(\hbar^{1 / 18}\right) .
\end{aligned}
$$

Thus, Theorems 4.1 and 4.4 can be supplemented by the following result.

Theorem 5.2. Under the assumptions as above, let an eigenvalue $\lambda=\lambda(\hbar)$ of the operator $H_{\hbar}$ belong to a neighborhood of $\lambda_{0}$. Then, necessarily, condition (5.2) is satisfied with the numbers $\varphi_{ \pm}\left(x_{0} ; \lambda\right)$ and $p\left(x_{0}, \lambda\right)$ defined by (4.2) and (5.1), respectively. All assertions ( for $x_{1}=x_{0}$ ) of Theorem 4.4 about the corresponding normalized eigenfunction $\psi(x ; \lambda, \hbar)$ are true with the constants $c_{ \pm}(\lambda, \hbar)$ whose absolute values are determined by formulas (5.3), (5.4), and (5.5).

Remark 5.3. If the functions $v^{\prime}(x)$ and $v^{\prime \prime}(x)$ are bounded in a neighborhood of the point $x_{0}$, then even in the case where $v\left(x_{0}+0\right) \neq v\left(x_{0}-0\right)$ estimates of all remainders are the same as in $\S 4$. Thus, we have $O(\hbar)$ in (5.2) and $O\left(\hbar^{1 / 6}\right)$ in (5.3) $-(5.5)$.

Remark 5.4. Under the above assumptions, let $v\left(x_{0}+0\right)=v\left(x_{0}-0\right)$. Then all conclusions of Proposition 5.1 remain true although the function $v^{\prime}(x)$ is not required to be continuous at $x_{0}$. In particular, we see that the jumps of derivatives of the function $v(x)$ at $x_{0}$ are inessential.

5.3. We consider an explicit example:

$$
v(x)=a_{+}+v_{+} x^{\alpha_{+}} \text {for } x>0 \text { and } v(x)=a_{-}+v_{-}|x|^{\alpha_{-}} \text {for } x<0,
$$

where $v_{ \pm}>0$ and $\alpha_{ \pm}>0$. Then all $\lambda>\max \left\{a_{+}, a_{-}\right\}$are noncritical, the equation $v(x)=\lambda$ has two solutions $x_{+}>0, x_{-}<0$, and $\left(x_{-}, x_{+}\right)$is a potential well. The point $x_{0}=0$ might be singular, and $p(0, \lambda)=\left(\lambda-a_{-}\right)^{1 / 4}\left(\lambda-a_{+}\right)^{-1 / 4}$. 
For the potentials (5.6), the integrals in (4.4) and (4.18) can be calculated in terms of the beta function B. Observe that $x_{+}=\left(\lambda v^{-1}\right)^{1 / \alpha}$ if $v(x)=v x^{\alpha}$ for $x>0$. For the integrals over $\left(0, x_{+}\right)$, we have

$$
\int_{0}^{x_{+}}\left(\lambda-v x^{\alpha}\right)^{1 / 2} d x=\lambda^{1 / 2}(\lambda / v)^{1 / \alpha} \alpha^{-1} \mathrm{~B}(3 / 2,1 / \alpha)
$$

and

$$
\int_{0}^{x_{+}}\left(\lambda-v x^{\alpha}\right)^{-1 / 2} d x=\lambda^{-1 / 2}(\lambda / v)^{1 / \alpha} \alpha^{-1} \mathrm{~B}(1 / 2,1 / \alpha) .
$$

The integrals over $\left(x_{-}, 0\right)$ can be calculated quite similarly.

It follows that the quantization condition (5.2) is satisfied with

$$
\begin{aligned}
& \varphi_{+}(0, \lambda)=\lambda_{+}^{1 / 2+1 / \alpha_{+}} v_{+}^{-1 / \alpha_{+}} \alpha_{+}^{-1} \mathrm{~B}\left(3 / 2,1 / \alpha_{+}\right), \\
& \varphi_{-}(0, \lambda)=\lambda_{-}^{1 / 2+1 / \alpha_{-}} v_{-}^{-1 / \alpha_{-}} \alpha_{-}^{-1} \mathrm{~B}\left(3 / 2,1 / \alpha_{-}\right),
\end{aligned}
$$

where $\lambda_{ \pm}=\lambda-a_{ \pm}$. In particular, if $a_{+}=a_{-}=: a$, then the Bohr-Sommerfeld quantization condition reads

$$
\begin{aligned}
& (\lambda-a)^{1 / 2+1 / \alpha_{+}} v_{+}^{-1 / \alpha_{+}} \alpha_{+}^{-1} \mathrm{~B}\left(3 / 2,1 / \alpha_{+}\right) \\
& \quad+(\lambda-a)^{1 / 2+1 / \alpha_{-}} v_{-}^{-1 / \alpha_{-}} \alpha_{-}^{-1} \mathrm{~B}\left(3 / 2,1 / \alpha_{-}\right)=\pi \hbar(n+1 / 2)+O\left(\hbar^{2}\right) .
\end{aligned}
$$

Plugging expressions (5.7) and (5.8) into (1.4), we also obtain

$$
K_{\mathrm{cl}}(\lambda)=\frac{\lambda_{+}^{1 / 2+1 / \alpha_{+}} v_{+}^{-\alpha_{+}} \alpha_{+}^{-1} \mathrm{~B}\left(3 / 2,1 / \alpha_{+}\right)+\lambda_{-}^{1 / 2+1 / \alpha_{-}} v_{-}^{-\alpha_{-}} \alpha_{-}^{-1} \mathrm{~B}\left(3 / 2,1 / \alpha_{-}\right)}{\lambda_{+}^{-1 / 2+1 / \alpha_{+}} v_{+}^{-\alpha_{+}} \alpha_{+}^{-1} \mathrm{~B}\left(1 / 2,1 / \alpha_{+}\right)+\lambda_{-}^{-1 / 2+1 / \alpha_{-}} v_{-}^{-\alpha_{-}} \alpha_{-}^{-1} \mathrm{~B}\left(1 / 2,1 / \alpha_{-}\right)} .
$$

Observe that Theorems 4.1 and 4.4 can be applied to the potential (5.6) if $a_{+}=a_{-}$ and $\alpha_{ \pm} \geq 2$. If $a_{+}=a_{-}$but $\alpha_{ \pm} \in[1,2)$, then we can use Remark 5.4] in this case $O\left(\hbar^{2}\right)$ in (5.9) should be replaced by $O\left(\hbar^{5 / 3}\right)$. If $a_{ \pm}$are arbitrary and $\alpha_{ \pm} \geq 1$, then the conditions of Theorem 5.2 are satisfied. Moreover, by Remark 5.3 in the case where $\alpha_{ \pm} \geq 2$, the estimates of the remainders can be improved. Finally, we note that if $\alpha_{j}<1$, then $v^{\prime \prime} \notin L^{1}(-\delta, \delta)$, so that the semiclassical approximation does not work directly (even for $a_{+}=a_{-}$) although all formulas above remain meaningful.

5.4. Now we briefly consider the problem on the half-axis. We suppose that equation (1.1) is satisfied for $x \geq 0, \psi \in L^{2}\left(\mathbb{R}_{+}\right)$, and $\psi(0)=0$. Assumptions 2.1 and 2.2 should be modified slightly. Namely, we assume that the equation $v(x)=\lambda$ has only one solution $x_{+}=x_{+}(\lambda)$ and $v^{\prime}\left(x_{+}\right)>0$, so that $\left(0, x_{+}\right)$is a potential well. We suppose that the limit of $v(x)$ as $x \rightarrow 0$ exists and that the functions $v^{\prime}(x)$ and $v^{\prime \prime}(x)$ are bounded in a neighborhood of $x=0$. Then the results of Theorem 2.5 about the solution $u_{+}(x)$ of equation (1.1) are true for all $x \geq 0$. In particular, from formula (2.19) it follows that

$$
\begin{aligned}
u_{+}(0 ; \lambda, \hbar)= & \pi^{1 / 2} \hbar^{1 / 6}(\lambda-v(0))^{-1 / 4} \\
& \times \sin \left(\hbar^{-1} \int_{0}^{x_{+}(\lambda)}(\lambda-v(x))^{1 / 2} d x+\pi / 4\right)+O\left(\hbar^{7 / 6}\right) .
\end{aligned}
$$

Since $\psi(x ; \lambda, \hbar)=c_{+} u_{+}(x ; \lambda, \hbar)$, this yields the quantization condition

$$
\int_{0}^{x_{+}(\lambda)}(\lambda-v(x))^{1 / 2} d x=\pi \hbar(n+3 / 4)+O\left(\hbar^{2}\right),
$$

where $n=n(\lambda, \hbar)$ is an integer. 
Consider the boundary condition $\psi^{\prime}(0)=b \psi(0), b=\bar{b}$. By (2.21), we have

$$
\begin{aligned}
u_{+}^{\prime}(0 ; \lambda, \hbar)=- & \pi^{1 / 2} \hbar^{-5 / 6}(\lambda-v(0))^{1 / 4} \\
& \times \cos \left(\hbar^{-1} \int_{0}^{x_{+}(\lambda)}(\lambda-v(x))^{1 / 2} d x+\pi / 4\right)+O\left(\hbar^{1 / 6}\right) .
\end{aligned}
$$

Comparing this with (5.10), we see that the value of $u_{+}(0 ; \lambda, \hbar)$ is inessential, so that the quantization condition looks like this:

$$
\int_{0}^{x_{+}(\lambda)}(\lambda-v(x))^{1 / 2} d x=\pi \hbar(n+1 / 4)+O\left(\hbar^{2}\right) .
$$

It does not depend on $b$.

The other results of $\S 4$ can also be extended naturally to the problem on the half-axis.

\section{ACKNOWLEDGMENT}

I thank D. Robert for a discussion of papers on microlocal analysis.

\section{REFERENCES}

[1] V. M. Babich and V. S. Buldyrev, Asymptotic methods in diffraction problems of short-length waves. The method of canonical problems, Nauka, Moscow, 1972; English transl., Short-wavelength diffraction theory. Asymptotic methods, Springer Ser. on Wave Phenomena, vol. 4, Springer-Verlag, Berlin, 1991. MR0426630 (54:14569) MR1245488 (94f:78004)

[2] M. V. Fedoryuk, Asymptotic methods for linear ordinary differential equations, Nauka, Moscow, 1983. (Russian) MR0732787 (85m:34001)

[3] V. P. Maslov and M. V. Fedoryuk, Quasiclassical approximation for the equations of quantum mechanics, Nauka, Moscow, 1976; English transl., Semiclassical approximation in quantum mechanics, Math. Phys. Appl. Math., vol. 7. Contemp. Math., vol. 5, D. Reidel, Dordrecht-Boston, MA, 1981. MR0461590 (57:1575) MR0634377 (84k:58226)

[4] B. Helffer, A. Martinez, and D. Robert, Ergodicité et limite semi-classique, Comm. Math. Phys. 109 (1987), 313-326. MR0880418 (88e:81029)

[5] B. Helffer and D. Robert, Puits de potentiel généralisés et asymptotique semi-classique, Ann. Inst. H. Poincaré Phys. Théor. 41 (1984), no. 3, 291-331. MR0776281 (86m:81049)

[6] L. D. Landau and E. M. Lifshits, Theoretical physics. Vol. 1. Mechanics, Fizmatlit, Moscow, 1958; English transl., Course of theoretical physics. Vol. 1. Mechanics, Pergamon Press, Oxford, 1976. MR0102191 (21:985) MR0475051 (57:14673)

[7] F. W. J. Olver, Asymptotics and special functions, Acad. Press, New York-London, 1974. MR0435697 (55:8655)

[8] B. Simon, Semiclassical analysis of low lying eigenvalues. II. Tunneling, Ann. of Math. (2) 120 (1984), 89-118. MR0750717 (87h:81045a)

Irmar, Université de Rennes I, Campus de Beaulieu, 35042 Rennes Cedex, France

E-mail address: yafaev@univ-rennes1.fr

Received 5/AUG/2010

Originally published in English 